\title{
CALAMIDADES, INTENCIONALIDADES Y DAÑOS
}

\author{
Carlos Thiebaut \\ Universidad Carlos III de Madrid
}

RESUMEN. Se analizan tres rasgos —intencionalidad, posibilidades alternativas, control- de la atribución de responsabilidad en contextos negativos o calamidades, en la terminología de EGV. Se argumenta que en esos contextos la perspectiva en primera persona no tiene prevalencia y que la tiene, por el contrario, la semántica moral detallada de lo que consideramos daños.

Palabras clave: Ernesto Garzón Valdés, intencionalidad, responsabilidad, daño.

ABSTRACT. Intentionality, alternative possibilities and control are analysed as three basic traits in responsibility attribution in negative contexts, or calamities in EGV's terminology. In these contexts, first-person perspectives are not taken as prevalent and a determining role is played by the detailed moral semantics of what is taken to be harmful.

Keywords: Ernesto Garzón Valdés, intentionality, responsability, harmful. 


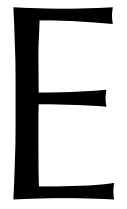

n las últimas décadas del siglo pasado creció la conciencia de las aterradoras realidades que lo habían jalonado y adquirió forma una sensibilidad cultural ante lo negativo de la experiencia humana que está extendiendo su sombra hasta ahora mismo. Como parte de esa sensibilidad, e intentando darle forma conceptual, ha crecido también una anegadora literatura sobre el mal y los males del siglo pasado que, no obstante, está teniendo un efecto ambiguo: por una parte, ha tematizado una conciencia de horror - $\mathrm{y}$ a veces ha contribuido a extenderla - ante lo que pudieran tener en común la larga lista de nombres propios con el que nombramos el mal (Auswitzch, Hiroshima, Sudáfrica, los desaparecidos, el 11-M, etc.); por otra, ha desplazado al plano de la reflexión filosófica, cuando no estrictamente metafísica, la interpretación de una posible raíz común de tan larga y diversa experiencia del mal: el fuste torcido de la humanidad, el mal radical, o la impenitente culpabilidad de permanecer en un estado de minoría de edad pueden ser algunas explicaciones ilustradas de tal raíz común, a las que cabría añadir la antropología ambiguamente pesimista de FREUD o la prevalencia de la voluntad y del poder en las interpretaciones postilutradas en la estela de NIETZSCHE. Por lo primero parece haberse extendido un tipo de sensibilidad reactiva que agudiza percepciones y dispara denuncias; por lo segundo, no obstante, a veces se ha dejado en una confusa nube conceptual aquello que suscita repudio y condena. El riesgo es el de un déficit conceptual que acabe por dejar ciegas aquellas percepciones y rechazos no menos que inútilmente vacíos los conceptos con los que comprendemos normativamente las acciones humanas. La tarea filosófica sería, precisamente, la de atar la antes mencionada conciencia del horror con los conceptos adecuados que den cuenta de los repudios, las denuncias y las condenas. Tal vez lo primero que cabe sugerir es que, para ello, hay que dejar, provisional y cautelosamente, de lado el mismo concepto de mal y emplear otras categorías para la dicha tarea filosófica. Con todas las cautelas filosóficas, tal vez, y en otro momento, ese concepto pueda encontrar un lugar significativo si se prescinde de sus connotaciones teológicas o de su cargada fuerza simbólica religiosa.

EGV ha acotado conceptualmente algunos de los males del siglo pasado y del presente (algunos de los dichos y otros) bajo la categoría de calamidad, por la que entiende las desgracias, desastres o miserias que resultan de acciones humanas intencionales y ha reservado el término catástrofe para designar aquellas desgracias, desastres o miserias provocados por causas naturales que escapan al control humano. «Es obvio - dice- que, si se acepta esta propuesta terminológica, las calamidades son evitables y las catástrofes no. En el caso de las primeras cabe hablar de responsabilidad normativa (moral o jurídica); en la segundas no» ${ }^{1}$. Estas líneas aceptan esta propuesta de EGV y sólo intentan interpretarla en otro lenguaje filosófico y, tal vez, plantear un problema respecto a algo que pudiera —erróneamente, a mi entender- entenderse que esa propuesta significa. El criterio de diferenciación entre calamidades y catástrofes parece tener, para EGV, una triple apoyatura: la de la intencionalidad, la del poder haber actuado de manera distinta a como se hizo (lo que se ha llamado el principio de las posibilidades alternativas) y la del control sobre las propias acciones. Estos tres elementos conceptuales han constituido, en efecto, y en maneras diversas, el entramado conceptual sobre el que se apoyan — tanto en la discusión clásica como en la

${ }^{1}$ Garzón Valdés, 2004: Calamidades, Barcelona: Gedisa, 2004, p. 12 
contemporánea- las atribuciones de responsabilidad y, en las discusiones de la metafísica moral, el supuesto de la libertad humana. En diversas maneras, las discusiones contemporáneas sobre el libre albedrío insisten, con diferentes acentos, sobre esos tres elementos o, al menos, sobre los dos últimos, el principio de las posibilidades alternativas y el control sobre las acciones. Tal vez, no obstante, el de intencionalidad sea un concepto más borroso. Probablemente, el uso, que remite a la tradición filosófica, que del concepto de intencionalidad hace EGV tiene un sentido de acotación inicial: nos hacemos responsables de aquello que hacemos de manera no casual ni torpe, de aquello que advertimos y no de que lo provocamos inadvertidamente. Por eso —en nuestras intuiciones cotidianas y en determinados usos específicos regidos por normas, como los jurídicos-el de intencionalidad parece un concepto imprescindible; pero, por otra, definir en la actitud de segunda o de tercera persona la intencionalidad de un acto, que es una característica de la agencia en actitud de primera persona, plantea en las interacciones mismas no pocos problemas. Cabe decir, incluso, que ese problema es el que está en la raíz de nuestras maneras de intentar comprender y de intentar regular las interacciones humanas. La pregunta «¿qué quiso hacer $\mathrm{X}$ con la acción A?» está a la base de cómo podemos conjuntar la autonomía del agente con las respuestas que sus actos nos suscitan o nos reclaman.

La cosa no se hace más clara, sino más oscura, cuando las dichas condiciones de atribución de responsabilidad —intención, posibilidades alternativas y control— se trasladan desde contextos positivos de elección - deliberar y elegir el bien que se busca- a contextos negativos - atribuir responsabilidad por un acto que consideramos repudiable- . A diferencia de los contextos positivos, en los que cabe pensar que el lugar de los otros es secundario o subsiguiente a la valoración que de la acción hace el sujeto (lo que no quiere decir que sea irrelevante ni para él ni para los otros), en los contextos negativos ese lugar (el de otros, el nuestro) es inmediato y determinante. La segunda y tercera persona del singular y la primera del plural son, precisamente, los puntos de partida de la comprensión misma de la acción y el punto de arranque del juicio que merezca. Parecería inicialmente que también en estos casos se sostienen los tres criterios que, para ellos, propone EGV: repudiar, condenar un mal o responsabilizarle a alguien de él (ya, de una calamidad) significa indicar que lo que hace o hizo tuvo intención de hacerlo, que pudo no haberlo hecho y que controlaba las causas y/o los efectos de lo que hacía. Si, por el contrario, le exoneramos al actor de la responsabilidad por tal hecho es porque - decimos - no quería hacer lo que hizo, porque no pudo evitar hacerlo y/o porque no podía controlar lo que hacía. Esta descripción general parece, en efecto, casar adecuadamente con nuestras intuiciones morales también en los contextos negativos, pero notemos que en ellos se hacen más inquietantes los perfiles borrosos de nuestros tres criterios de responsabilidad y que con frecuencia nuestras justificaciones y exoneraciones juegan tanto con esa borrosidad como con la no menor de decidir si, en un caso concreto, se dan los tres rasgos o los que parezcan pertinentes en nuestra condena. La gramática de nuestras excusas, como notó AusTin, está a la base de nuestra gramática moral o, al menos, tiene la fuerza de ponerla en evidencia.

En el libro Calamidades se dan claras muestras de las constricciones de nuestras excusas, en concreto, de cuándo las excusas inicialmente aducidas no pueden considerarse válidas. Si aceptar una excusa es poner en evidencia los perfiles de nuestra gramática moral (por vía, por ejemplo, de restringir nuestras emociones reactivas — por decirlo 
con STRAWSON- o de aclarar las condiciones de ejercicio de nuestros juicios normativos), rechazar una excusa aducida ante una condena hace más precisos, por más exigentemente detallados y más justificados, los enunciados normativos de esa gramática. No siempre realizar una condena equivale a rechazar una excusa, pero, al menos, el rechazo de una excusa hace conceptualmente más claras las razones de una condena. Siguiendo a EGV, veamos cómo pueden rechazarse las excusas en los contextos negativos que constituyen las calamidades y el papel que en ellas juegan la perspectiva del agente y la de una perspectiva de segunda, tercera persona o la de la primera del plural. En primer lugar, es probable que el que el agente indique que no pudo actuar de manera distinta a como lo hizo sea, en efecto, como indica EGV, el elemento más claro en su defensa. El que no hubiera posibilidades alternativas a lo que hizo (porque el agente estaba constreñido a hacerlo) es otra manera de indicar que le era necesario hacerlo, que le era inevitable. El mostrar que tal no es el caso - porque no era de hecho el caso, o, recursivamente, porque el agente erró en sus creencias respecto a lo que podía o no podía hacerse- ha constituido el esfuerzo repetido en la historia de las maneras en las que se ha ido arrancando de la esfera de los males inevitables algunos comportamientos y acciones que juzgamos, más bien, calamidades o daños. Por poner un ejemplo de esta recursiva responsabilidad doxástica, el principio de la obediencia debida fue argüido en la defensa de los acusados en Nüremberg y reiterado en múltiples ocasiones, desde las dictaduras latinoamericanas, la Guerra de Vietnam o las vejaciones de Abu-Graib. Rechazando lo que el agente tomaba como (justificadas) constricciones de lo que hizo - en el ejemplo, su creencia en que debía seguir las órdenes recibidas- es como se han ido afinando los criterios de nuestros repudios y condenas y parece que el punto crucial de este proceso es el antes señalado de indicar que los agentes son recursivamente responsables de las creencias erradas (como la de la incuestionablidad de la obediencia ciega en contextos bélicos) que tuvieran con respecto a lo que era el caso, a su incapacidad de haber tomado un rumbo alternativo a lo que hicieron o a las normas adecuadas sobre lo que juzgaban correcto en el juicio de lo que hicieron. Que se podía haber actuado a manera distinta como se hizo y que, consiguientemente, se podía haber creído que se podía actuar de manera distinta a lo que se hizo, parece, en efecto, un punto cero en la atribución de responsabilidad en contextos negativos.

Probablemente, y en segundo lugar, también el criterio del control sobre las propias acciones tenga rasgos similares a lo que se acaba de comentar, aunque alguna borrosidad más preocupante pueda empezar a metérsenos de por medio. Cabe que el agente argumente que no pudo controlar — porque era imprevisible, por ejemplo, dice EGV - los efectos de su acción. Ante tal excusa, podemos aplicar el mismo criterio recursivo antes indicado y señalar que, en determinado nivel de conocimientos (científicos, sociales, etc.) que se le suponen o se le pueden suponer razonablemente al agente, aducir ignorancia respecto a los efectos de sus actos refuerza, incluso, nuestra condena. Al igual que pudo saber, o que debía haber sabido, que había posibilidades alternativas a lo que hizo, cabe pensar que pudo prever, o debía haber previsto, los efectos de sus actos. No obstante, estamos ya ante una esfera de matices de más difícil apreciación, pues también sabemos que la gama de efectos no deseados de las acciones camina a la contra de la capacidad de control que el agente dice disponer sobre ellas. $\mathrm{Mu}$ chas veces, de haber sabido lo que resultaba de lo que iniciamos no lo habríamos hecho. 
Pero ese posible arrepentimiento no tiene por qué exonerarnos de nuestra responsabilidad ante lo hecho, algo que puede constatarse o argüirse en la actitud de segunda y de tercera persona que, como dijimos, es un elemento determinante de los contextos negativos; pero notemos, entonces, que la perspectiva del agente en primera persona respecto a su propio control sobre lo que hizo parece, perder relevancia. Contra lo que el agente pueda aducir como excusa — los efectos no deseados por él de su acto- - aducimos que lo hizo y que debía — recursivamente- haber sabido lo que hacía y qué efectos tendría. En este caso, el que «debía haberlo sabido» parece una condición doxástica y normativa que opera en paralelo con la que antes señalamos, a pesar de que al aplicarla seamos conscientes de que sus condiciones de aplicación («lo que razonablemente debería haberse sabido sobre los efectos de los actos») tengan límites borrosos.

Pero, probablemente, el problema se hace más borroso e inquietante aun cuando acudimos a la primera, y más englobante, característica de la responsabilidad sobre las propias acciones que es la intencionalidad ${ }^{2}$. Ciertamente, parecería una excusa radical el indicar que no quisimos hacer lo que hicimos y ese enunciado en primera persona, apoyado sobre la incuestionable autoridad que parecemos reclamar sobre nuestras creencias, deseos e intenciones se expresa, por ejemplo, cuando pedimos disculpas. No parece que, en este caso, podamos acudir al mecanismo recursivo al que antes apelamos. Porque la intencionalidad no permite ser objeto, en contextos negativos, de un argumento del tipo: «X no debía haber tenido la intención I» dado que el agente apela en su alegato, precisamente, a que no tenía tal intención. La autoridad del sujeto sobre sus estados mentales parece imponer, en este caso, una barrera en nuestras atribuciones recursivas. Cuando en un contexto negativo el agente se desliga de sus actos, el juicio condenatorio que éstos pudieran merecer ha de desligarse, también, de la apelación a la intencionalidad expresada por el agente que, como tal, nos es opaca. Ello no desarma, no obstante, la atribución de responsabilidad: que nos sea opaca la intencionalidad dicha no quiere decir que lo sean también sus actos; el agente es responsable de lo que bizo, con independencia de que diga ahora que no quiso hacerlo o de que no sabía lo que hacía. Sobre lo hecho apoyamos nuestro repudio del acto y nuestro rechazo de la posible excusa de que hizo lo que hizo de manera no intencional. Podemos, en contextos acotados e irrelevantes, aceptar la excusa, como cuando rompemos un objeto apreciado por nuestro anfitrión y aducimos la accidentalidad de lo sucedido; también, como sucede en determinados accidentes que resultan en muertes, aunque con mayor prudencia, podemos aceptar la no intencionalidad como excusa; no parece, no obstante, posible que casos especialmente relevantes, que requieren una sistemática falta de percepción y un sistema también sistemático de actos —recordemos el caso EICHMANNpodamos aceptar que lo hecho era accidental o no intencional. En casos así - de calamidades - la intencionalidad expresa del agente, aducida como excusa, nos parece no pertinente y los actos hablan por sí solos. Pudiéramos contraargüir, no obstante (pudiera hacerlo el agente), que lo que, entonces, está en cuestión no es tanto su intencionalidad, sino su veracidad. Y, en efecto, sospechamos de la veracidad del agente en con-

${ }^{2}$ Es interesante notar que en la literatura analítica sobre el concepto de responsabilidad — por ejemplo, en los trabajos de Harry FRANKFURT—, se suele entender aproximadamente por «control sobre las propias acciones» no tanto lo que hemos mencionado en el párrafo anterior, el control sobre los efectos, sino la capacidad de identificarse que tiene el agente con sus actos que aparece aquí ahora bajo la categoría de intencionalidad. 
textos en los que la apelación a ella va a contracorriente de nuestros juicios condenatorios con respecto al carácter condenable de lo que hizo. Pero, entonces, y de nuevo, es una condición doxástica — paralela a las anteriores_ la que pasa a primer plano: rechazamos que la relación que el agente condenable establece entre sus creencias y lo que es el caso sea la que de hecho se da entre esos términos (él reclamándose veraz, nosotros sospechando de esa veracidad). Una consecuencia filosófica de ello -un resultado externista, no internista- es que en contextos negativos el criterio de la intencionalidad no es tan determinante para dirimir responsabilidades como pudiera parecer que lo es en contextos positivos. Si en éstos, como decíamos, nuestras intenciones nos explican el carácter de nuestros actos y el significado que pueden tener para nosotros u otros, en los negativos acudir a la exoneración de no-haber-tenido-la-intención-de-X no parece restringir nuestras emociones reactivas o nuestros ponderados juicios de condena. Parecemos operar, entonces, con una cierta esquizofrenia: por una parte consideramos la intencionalidad un rasgo de las acciones humanas que introduce para su comprensión un conjunto de categorías normativas (señaladamente, las morales, pero también las jurídicas) y que nos permite considerarlas dignas de alabanza, mérito, etc; por otra parte, en contextos negativos podemos prescindir —o vernos forzados a prescindir - de la expresa declaración de intencionalidad del agente (y atribuirle intenciones distintas a las expresadas, o alguna patológica incomprensión respecto a ellas) y enfrentarla con el juicio negativo que nos merece lo que hizo. Cuándo y cómo prescindimos de la intencionalidad del agente parece ser la letra pequeña, pero cotidiana, de nuestra gramática moral.

El problema que estas borrosidades introducen en los contextos negativos de atribución de responsabilidad es que el fundamento de dicha atribución se ha desplazado desde el punto de vista del agente al de la segunda o tercera persona (y a la primera del plural). Es una definición, potencialmente externa al agente, de determinados actos como condenables lo que acaba por permitir la dicha atribución de responsabilidad; es, igualmente, la que permite fundamentar la atribución recursiva de lo errado de sus creencias (sobre lo que podía hacerse, sobre lo que debía creerse era adecuado, sobre lo que podía haberse previsto). Cabe sugerir que, en contextos negativos, es la definición de lo que sea un daño lo que determina ese su carácter negativo. Pero, entonces, el problema se desplaza a qué consideramos, y por qué, daños. El principio de MiLl es un principio formal (permitir todo lo que no daña) que, no obstante, permanece impreciso. Lo que consideramos daños —o calamidades— queda remitido a la semántica moral precisa de aquellos comportamientos o actos sobre otros que podemos ir argumentando que les lesionan, que les producen sufrimiento o que les hace padecer situaciones injustas. El catálogo de calamidades de EGV es parte de esa semántica moral, siempre abierta a ulteriores precisiones y al desentrañamiento de su estructura conceptual. 